\title{
APORTES A LA INVESTIGACIÓN GEOGRAFICA DESDE LA GEOGRAFÍA ELECTORAL EL CASO DE LA PROVINCIA DEL CHACO
}

\author{
Prof. Norma Monzón \\ Auxiliar Docente de Primera \\ Cátedra Técnicas en Geografía II \\ Instituto de Geografía - \\ Facultad de Humanidades - UNNE
}

\begin{abstract}
RESUMEN
En el presente trabajo pretendemos aportar conceptos teóricos referidos al estudio de la Geografía Electoral, a la vez que ilustramos con un caso de la Provincia del Chaco de las Elecciones para Gobernador en el año 2011. También deseamos destacar que el desarrollo de este abordaje de la Geografía Humana, su estudio y la formación de geógrafos electorales es escaso en Argentina. Presentamos las técnicas y métodos de estudio, así como las dificultades en la investigación electoral. Con lo cual, consideramos que este trabajo es un aporte al conocimiento de un aspecto de la Ciencia Geográfica
\end{abstract}

\section{PALABRAS CLAVES}

Geografía Electoral - Elecciones Provincia del Chaco - Comportamiento Electoral

\begin{abstract}
In the present work we intend to contribute with theoretical concepts related to the study of the Electoral geography, as well as show a reference case of Chaco's province election for governor in 2011. We also want to enhance that the development of this approaching to human geography, its study and the formation of electoral geographers is limited in Argentina. We present studies and methodologies techniques, as well as the difficulties during the electoral investigation. We consider this work to be a contribution to the knowledge of an aspect of the geographic science.
\end{abstract}

KEY WORDS: Electoral Geography-Chaco's Province Election-Electoral Behaviour

\section{INTRODUCCION}

La Geografía Electoral, en adelante G.E., estudia el proceso electoral que valida la continuidad del aparato estatal poniendo en función a sus autoridades. Las investigaciones de G.E. son muy cercanas a la Geografía Política, diferenciándose de esta por realizar estudios con énfasis en el análisis del comportamiento electoral de los ciudadanos en un momento electoral específico y en relación a un espacio geográfico determinado. Así mismo, desde la G.E. se realiza un abordaje ecológico al estudiar las interrelaciones existentes entre las variables sociodemográficas, culturales, económicas de la población con los resultados electorales, mediante lo cual se pretende entender que relación existe entre la manera de votar de los ciudadanos y las características mencionadas. Los resultados y conclusiones de las investigaciones de los geógrafos electorales se muestran en cartografías temáticas de inventario y de síntesis, gráficos y textos con conclusiones que permiten interpretar el comportamiento electoral de la ciudadanía, como así también aportan al conocimiento de la distribución de características específicas de la población. Con todos estos elementos resultantes de un estudio de G.E., la misma aporta insumos para otras investigaciones sociales, así como, mediante el análisis del voto se puede sectorizar la aprobación o descontento de los electores respecto de una acción de Gobierno, por lo cual la G.E. puede promover propuestas de solución en cuanto a la correcta aplicación de políticas públicas.

\section{ALGUNAS DEFINICIONES}

Bosque Sendra caracteriza a la G.E. como "...subdisciplina geográfica -muy minoritaria en cuanto a números de practicantes- como una de las partes de la Geografía Humana que más se aproxima a una verdadera ciencia "dura" semejante a cualquier ciencia experimental" (Bosque Sendra, 1988:13)

Por su parte, las autoras Silvia Gómez Tagle y María Eugenia Valdés definen a la G.E. como "un método cartográfico para definir el reparto regional de las fuerzas partidarias, la Geografía Electoral permite identificar las zonas donde tienen influencia los Partidos $y$, de esta manera, observar las relaciones entre las tendencias políticas y las características de la población que vive en esas zonas geográficas" (Gómez Tagle y Valdez, 2000:14) 
A su vez Johnston en 1987 publicó el "Diccionario de Geografía Humana" en el cual define a la G.E. en los siguientes términos

"GEOGRAFÍA ELECTORAL: Estudio de los aspectos geográficos de la organización, realización y resultados de las elecciones. A principios de siglo se publicaron algunos artículos pioneros en Geografía Electoral (existe una fuerte tradición francesa), pero la bibliografía no es muy abundante y casi toda es de fecha reciente. Pocos profesionales de la Geografía Humana se dedican al estudio de los procesos electorales, y no abundan los contactos entre esta subdisciplina y la Geografía Política.

Hay cinco grandes áreas de estudio en Geografía Electoral.

a) La organización espacial de las elecciones, con especial referencia a la definición de circunscripciones.

b) Las variaciones espaciales en las pautas del voto, más las relaciones entre éstas y otras características poblacionales, en concreto la clase social;

c) La influencia de los factores ambientales y espaciales en las decisiones sobre el voto,

d) Las estructuras espaciales de representación producidas al traducirse los votos en escaños en un parlamento y organismo similar

e) Las variaciones en el espacio del reparto del poder y de la implementación de las políticas que reflejan las pautas de representación" (Johnston, 1987:52)

\section{MATERIALES Y METODOS}

Todo lo dicho anteriormente, implica el desarrollo de métodos y técnicas variadas atribuyendo a la G.E. una característica interdisciplinaria.

\section{Métodos y técnicas aplicadas en Geografía Electoral: puente entre lo cualitativo y lo} cuantitativo.

En el caso de la investigación en G.E. la combinación del estudio de los factores individuales y contextuales que se identifican en la toma de decisión del elector al momento de votar, nos demanda realizar análisis cuali-cuantitativos.

Con este fundamento, presentamos a la G.E. como un puente que une con su estudio técnicas cuantitativas y cualitativas ligadas por un tronco en común: el espacio geográfico (figura 1).

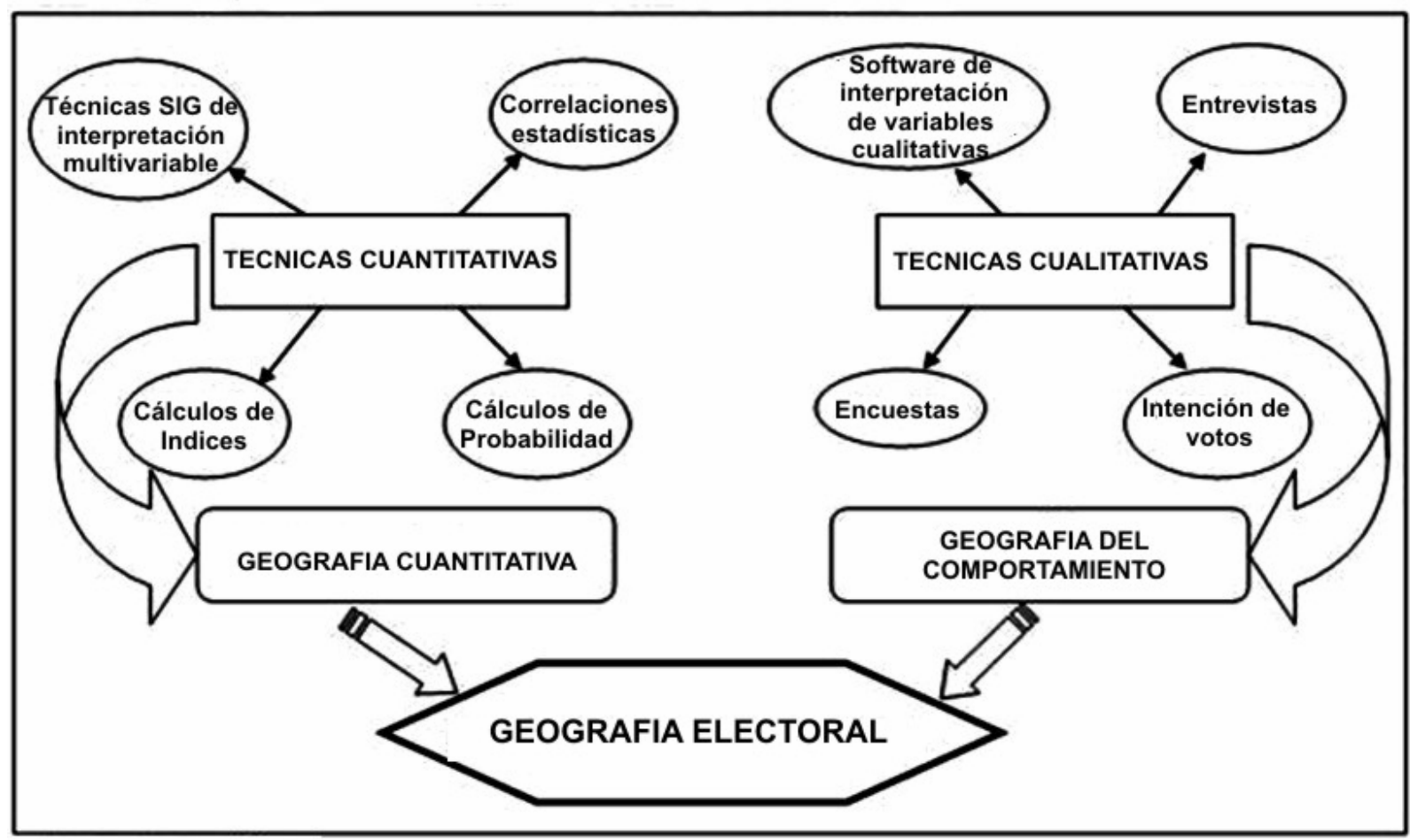

Fuente: Elaboración propia 
Destacamos que el caudal de información estadística, esto es, tanto de los resultados electorales como de datos sociodemográficos que se estudia de manera interrelacionada en G.E.. demanda la utilización de softwards (los más utilizados son los del entorno de los Sistemas de Información Geográfica -- SIG), que permiten interrelacionar múltiples variables entre sí (análisis multivariado), para poder trabajar con modelos y arribar a conclusiones que se aproximen al entendimiento del comportamiento electoral. Este trabajo cuantitativo lo explica Santiago Quesada en su artículo "La teoría de los sistemas y la Geografía Humana" del cual rescatamos en siguiente párrafo "Si su tarea ya sea planificador, investigador o docente- consiste precisamente en conocer y explicar las diferencias, discontinuidades, relaciones y tendencias de un país o región, o las interrelaciones en un grupo social, entonces se encontrará ante un problema complejo y de difícil resolución. Pero, en todo caso, se encontrará ante un problema clave de la ciencia social, en general, y de la geografía humana en particular. Es este un problema complejo por el gran número de elementos que inciden en el comportamiento de un grupo social en el espacio, lo cual hace que sea difícil establecer leyes y teorías; de hecho, sólo a partir del empleo de técnicas cuantitativas en la denominada "Nueva Geografía" ha podido abordarse eficazmente el planteamiento de este problema; estos intentos implican el uso de técnicas que permitan la relación formal de múltiples variables, tales como las técnicas de computación" (Quesada, 1978:15)

\section{DISCUSION}

Como todo proceso, el aspecto electoral dentro de una sociedad bajo un régimen democrático, posee elementos y factores que lo componen y a la vez lo condicionan, el cual se entiende como el período transcurrido desde el cronograma de Elecciones emanado desde el Poder Ejecutivo (Provincial, Nacional o local, según la categoría de la Elección) y el cumplimiento del mismo: presentación y oficialización de listas de candidatos, organización de la localización de la mesas receptoras de votos, confección del Padrón Electoral, fiscalización de la jornada electoral, escrutinio de votos y publicación oficial del mismo. La competencia de la G.E corresponde al estudio geográfico de algunos de estos componentes. De manera particular al análisis de la distribución geográfica de los resultados electorales, en la categoría electiva que el investigador seleccione: Elecciones Nacionales de Presidente y Vice, Senadores Nacionales y Diputados Nacionales, Elecciones Provinciales, Gobernador y Vice, Diputados Provinciales y en algunas provincias Senadores Provinciales, y las Elecciones Municipales mediante las cuales se eligen Intendentes y Concejales en cada Municipio.

El estudio de estos elementos que se realiza desde la G.E. tiene que ver con el espacio que se considera en el escrutinio -nuevamente según opción del investigador- puede ser el País, las Provincias individualmente, o las Provincias divididas en Departamentos o Municipios, y a su vez, la unidad mínima de estudio espacial de la G.E. que son los Circuitos Electorales. Esta unidad espacial es muy válida pues nos otorga la posibilidad de estudiar en mayor detalle la distribución del escrutinio de votos para cada Partido Político. El espacio geográfico de la Provincia del Chaco está organizado en 155 Circuitos Electorales identificados de manera numérica o alfanumérica, según el siguiente detalle.

\begin{tabular}{|c|l|l|l|l|}
\hline & N $^{\circ}$.E. & \multicolumn{1}{|c|}{ DEPARTAMENTO } & DENOMINACIÓN del C.E. & \multicolumn{1}{|c|}{ MUNICIPIO } \\
\hline 1 & 0001 & San Fernando & Resistencia & Resistencia \\
\hline 2 & 0002 & San Fernando & Resistencia & Resistencia \\
\hline 3 & 0003 & San Fernando & Resistencia & Resistencia \\
\hline 4 & 0004 & San Fernando & Resistencia & Resistencia \\
\hline 5 & $0005 \mathrm{~A}$ & San Fernando & Resistencia & Resistencia \\
\hline 6 & $0005 B$ & San Fernando & Resistencia & Resistencia \\
\hline 7 & 0006 & San Fernando & Resistencia & Resistencia \\
\hline 8 & $0007 \mathrm{~A}$ & San Fernando & Resistencia & Resistencia \\
\hline 9 & $0007 \mathrm{~B}$ & San Fernando & Resistencia & Resistencia \\
\hline 10 & $0008 \mathrm{~A}$ & San Fernando & Resistencia & Resistencia \\
\hline 11 & $0008 \mathrm{~B}$ & San Fernando & Resistencia & Resistencia \\
\hline 12 & 0009 & San Fernando & Barranqueras & Barranqueras \\
\hline
\end{tabular}


Revista Geográfica Digital. IGUNNE. Facultad de Humanidades. UNNE. Año 10. № 20. Julio - Diciembre 2013. ISSN 1668-5180 Resistencia, Chaco

\begin{tabular}{|c|c|c|c|c|}
\hline 13 & 0010 & San Fernando & Barranqueras & Barranqueras \\
\hline 14 & $0011 \mathrm{~A}$ & San Fernando & Barranqueras & Barranqueras \\
\hline 15 & 0011B & San Fernando & Barranqueras & Barranqueras \\
\hline 16 & 0012 & San Fernando & Puerto Vilelas & Puerto Vilelas \\
\hline 17 & $0013 A$ & San Fernando & Resistencia & Resistencia \\
\hline 18 & 0013B & San Fernando & Resistencia & Resistencia \\
\hline 19 & 0014 & San Fernando & Resistencia & Resistencia \\
\hline 20 & 0015 & San Fernando & Resistencia & Resistencia \\
\hline 21 & $0016 \mathrm{~A}$ & San Fernando & Resistencia & Resistencia \\
\hline 22 & 0016B & San Fernando & Resistencia & Resistencia \\
\hline 23 & 0016C & San Fernando & Resistencia & Resistencia \\
\hline 24 & 0017 & San Fernando & Resistencia & Resistencia \\
\hline 25 & 0018 & San Fernando & Resistencia & Resistencia \\
\hline 26 & 0019A & San Fernando & Resistencia & Resistencia \\
\hline 27 & 0019B & San Fernando & Resistencia & Resistencia \\
\hline 28 & 0020 & San Fernando & Resistencia & Resistencia \\
\hline 29 & 0021 & San Fernando & Resistencia & Resistencia \\
\hline 30 & $0022 \mathrm{~A}$ & San Fernando & Fontana & Fontana \\
\hline 31 & 0022B & San Fernando & Fontana & Fontana \\
\hline 32 & 0023 & San Fernando & Fontana & Fontana \\
\hline 33 & 0024 & San Fernando & Resistencia & Resistencia \\
\hline 34 & 0025 & San Fernando & Resistencia & Resistencia \\
\hline 35 & 0026 & San Fernando & Basail & Basail \\
\hline 36 & 0027 & San Fernando & Resistencia & Resistencia \\
\hline 37 & 0028 & 1 de Mayo & Cmdte Benítez & Cmdte Benítez \\
\hline 38 & 0029 & Primero De Mayo & Margarita Belén & Margarita Belén \\
\hline 39 & 0030 & Primero De Mayo & Costa Ine & Margarita Belén \\
\hline 40 & 0031 & Primero De Mayo & Colonia San Miguel & Margarita Belén \\
\hline 41 & 0032 & Libertad & Puerto Tirol & Puerto Tirol \\
\hline 42 & 0033 & Libertad & Colonia Popular & Colonia Popular \\
\hline 43 & 0034 & Libertad & Laguna Blanca & Laguna Blanca \\
\hline 44 & 0035 & Libertad & Fortín Cardozo & Puerto Tirol \\
\hline 45 & 0036 & Libertad & General Obligado & Puerto Tirol \\
\hline 46 & 0037 & General Dónovan & Lapachito & Lapachito \\
\hline 47 & 0038 & General Dónovan & Makalle & Makalle \\
\hline 48 & 0039 & General Dónovan & La Verde & La Verde \\
\hline 49 & 0040 & General Dónovan & La Escondida & La Escondida \\
\hline 50 & 0041 & Sargento Cabral & Colonia Elisa & Colonia Elisa \\
\hline 51 & 0042 & Sargento Cabral & Capitán Solari & Capitán Solari \\
\hline 52 & 0043 & Sargento Cabral & Colonias Unidas & Colonias Unidas \\
\hline 53 & 0044 & Sargento Cabral & Las Garcitas & Las Garcitas \\
\hline 54 & 0045 & Pcia. de La Plaza & Santa Elena & Pcia. de La Plaza \\
\hline
\end{tabular}


Revista Geográfica Digital. IGUNNE. Facultad de Humanidades. UNNE. Año 10. № 20. Julio - Diciembre 2013. ISSN 1668-5180 Resistencia, Chaco

\begin{tabular}{|c|c|c|c|c|}
\hline 55 & 0046 & Pcia. de La Plaza & El Curundu & Pcia. de La Plaza \\
\hline 56 & 0047 & Pcia. de La Plaza & Presidencia De La Plaza & Pcia. de La Plaza \\
\hline 57 & 0048 & Pcia. de La Plaza & Fortín Aguilar & Pcia. de La Plaza \\
\hline 58 & 0049 & Bermejo & Isla del Cerrito & Isla del Cerrito \\
\hline 59 & 0050 & Bermejo & Cancha Larga & La Leonesa \\
\hline 60 & 0051 & Bermejo & Laguna Patos & La Leonesa \\
\hline 61 & 0052 & Bermejo & Las Palmas & Las Palmas \\
\hline 62 & 0053 & Bermejo & La Leonesa & La Leonesa \\
\hline 63 & 0054 & Bermejo & General Vedia & General Vedia \\
\hline 64 & 0055A & Bermejo & Puerto Bermejo & Puerto Bermejo \\
\hline 65 & 0055B & Bermejo & Puerto Eva Perón & Puerto Eva Perón \\
\hline 66 & 0056 & Bermejo & El Retiro & General Vedia \\
\hline 67 & 0057 & Bermejo & Lapacho & La Leonesa \\
\hline 68 & 0058 & Lib. General San Martín & Selvas del Río de Oro & La Eduvigis \\
\hline 69 & 0059 & Lib. General San Martín & La Eduvigis & La Eduvigis \\
\hline 70 & 0060 & Lib. General San Martín & Pampa Almirón & Pampa Almirón \\
\hline 71 & 0061 & Lib. General San Martín & Campo El Bermejo & Laguna Limpia \\
\hline 72 & 0062 & Lib. General San Martín & Gral José de San Martín & Gral José de San Martín \\
\hline 73 & 0063 & Lib. General San Martín & Gral José de San Martín & Gral José de San Martín \\
\hline 74 & 0064 & Lib. General San Martín & Presidencia Roca & Presidencia Roca \\
\hline 75 & 0065 & Lib. General San Martín & Pampa Del Indio & Pampa Del Indio \\
\hline 76 & 0066 & Lib. General San Martín & Laguna Limpia & Laguna Limpia \\
\hline 77 & 0067 & Lib. General San Martín & Ciervo Petiso & Ciervo Petiso \\
\hline 78 & 0068 & Tapenagá & Cote Lai & Cote Lai \\
\hline 79 & 0069 & Tapenagá & La Sabana & Charadai \\
\hline 80 & 0070 & Tapenagá & Charadai & Charadai \\
\hline 81 & 0071 & Tapenagá & Horquilla & Charadai \\
\hline 82 & 0072 & Tapenagá & Haumonia & Charadai \\
\hline 83 & 0073 & San Lorenzo & Pozo Colorado & Villa Berthet \\
\hline 84 & 0074 & San Lorenzo & Villa Berthet & Villa Berthet \\
\hline 85 & 0075 & San Lorenzo & Samuhu & Samuhu \\
\hline 86 & 0076 & Mayor L. J. Fontana & Enrique Urien & Enrique Urien \\
\hline 87 & 0077 & Mayor L. J. Fontana & Villa Ángela & Villa Ángela \\
\hline 88 & 0078 & Mayor L. J. Fontana & Villa Ángela & Villa Ángela \\
\hline 89 & 0079 & Mayor L. J. Fontana & Villa Ángela & Villa Ángela \\
\hline 90 & 0080 & Mayor L. J. Fontana & Coronel Du Graty & Coronel Du Graty \\
\hline 91 & 0081 & Mayor L. J. Fontana & Mesón De Fierro & Coronel Du Graty \\
\hline 92 & 0082 & Mayor L. J. Fontana & Villa Ángela & Villa Ángela \\
\hline 93 & 0083 & O' Higgins & Colonia D. Matheu & San Bernardo \\
\hline 94 & 0084 & O' Higgins & San Bernardo & San Bernardo \\
\hline 95 & 0085 & O' Higgins & La Clotilde & La Clotilde \\
\hline 96 & 0086 & O' Higgins & La Tigra & La Tigra \\
\hline
\end{tabular}


Revista Geográfica Digital. IGUNNE. Facultad de Humanidades. UNNE. Año 10. № 20. Julio - Diciembre 2013. ISSN 1668-5180 Resistencia, Chaco

\begin{tabular}{|c|c|c|c|c|}
\hline 97 & 0087 & Cdte. Fernández & Pcia Roque Saenz Peña & Pcia Roque Saenz Peña \\
\hline 98 & 0088 & Cdte. Fernández & Pcia Roque Saenz Peña & Pcia Roque Saenz Peña \\
\hline 99 & 0089 & Cdte. Fernández & Pcia Roque Saenz Peña & Pcia Roque Saenz Peña \\
\hline 100 & 0090 & Cdte. Fernández & Pcia Roque Saenz Peña & Pcia Roque Saenz Peña \\
\hline 101 & 0091 & Cdte. Fernández & Pcia Roque Saenz Peña & Pcia Roque Saenz Peña \\
\hline 102 & 0092 & Cdte. Fernández & Pcia Roque Saenz Peña & Pcia Roque Saenz Peña \\
\hline 103 & 0093 & Cdte. Fernández & Pcia Roque Saenz Peña & Pcia Roque Saenz Peña \\
\hline 104 & 0094 & Cdte. Fernández & Pcia Roque Saenz Peña & Pcia Roque Saenz Peña \\
\hline 105 & 0095 & Cdte. Fernández & Pcia Roque Saenz Peña & Pcia Roque Saenz Peña \\
\hline 106 & 0096 & Cdte. Fernández & Pcia Roque Saenz Peña & Pcia Roque Saenz Peña \\
\hline 107 & 0097 & Cdte. Fernández & Pcia Roque Saenz Peña & Pcia Roque Saenz Peña \\
\hline 108 & 0098 & Quitilipi & Quitilipi & Quitilipi \\
\hline 109 & 0099 & Quitilipi & Quitilipi & Quitilipi \\
\hline 110 & 0100 & Quitilipi & Quitilipi & Quitilipi \\
\hline 111 & 0101 & Quitilipi & Quitilipi & Quitilipi \\
\hline 112 & 0102 & 25 de Mayo & Machagai & Machagai \\
\hline 113 & 0103 & 25 de Mayo & Machagai & Machagai \\
\hline 114 & 0104 & 25 de Mayo & Machagai & Machagai \\
\hline 115 & 0105 & 25 de Mayo & Machagai & Machagai \\
\hline 116 & 0106 & 25 de Mayo & Machagai & Machagai \\
\hline 117 & 0107 & Maipú & Tres Isletas & Tres Isletas \\
\hline 118 & 0108 & Maipú & Tres Isletas & Tres Isletas \\
\hline 119 & 0109 & Maipú & Tres Isletas & Tres Isletas \\
\hline 120 & 0110 & Independencia & Pampa del Regimiento & Avia Terai \\
\hline 121 & 0111 & Independencia & Napenay & Napenay \\
\hline 122 & 0112 & Independencia & Avia Terai & Avia Terai \\
\hline 123 & 0113 & Independencia & Campo Largo & Campo Largo \\
\hline 124 & 0114 & Independencia & Colonia José Mármol & Campo Largo \\
\hline 125 & 0115 & General Belgrano & Corzuela & Corzuela \\
\hline 126 & 0116 & General Belgrano & El Loro Blanco & Corzuela \\
\hline 127 & 0117 & 9 De Julio & Las Breñas & Las Breñas \\
\hline 128 & 0118 & 9 De Julio & Las Breñas & Las Breñas \\
\hline 129 & 0119 & Chacabuco & Charata & Charata \\
\hline 130 & 0120 & Chacabuco & Charata & Charata \\
\hline 131 & 0121 & 12 de Octubre & El Palmar & General Pinedo \\
\hline 132 & 0122 & 12 de Octubre & General Pinedo & General Pinedo \\
\hline 133 & 0123 & 12 de Octubre & Pampa Landriel & General Pinedo \\
\hline 134 & 0124 & 12 de Octubre & General Capdevila & General Capdevila \\
\hline 135 & 0125 & 12 de Octubre & Gancedo & Gancedo \\
\hline 136 & 0126 & 2 de Abril & Hermoso Campo & Hermoso Campo \\
\hline 137 & 0127 & 2 de Abril & Zuberbuhler & Hermoso Campo \\
\hline 138 & 0128 & Fray J. Sta M. de Oro & Santa Sylvina & Santa Sylvina \\
\hline
\end{tabular}


Revista Geográfica Digital. IGUNNE. Facultad de Humanidades. UNNE. Año 10. № 20. Julio - Diciembre 2013. ISSN 1668-5180 Resistencia, Chaco

\begin{tabular}{|l|l|l|l|l|}
139 & 0129 & Fray J. Sta M. de Oro & Chorotis & Chorotis \\
\hline 140 & 0130 & Almirante Brown & Concepción del Bermejo & Concepción del Bermejo \\
\hline 141 & 0131 & Almirante Brown & Pampa del Infierno & Pampa del Infierno \\
\hline 142 & 0132 & Almirante Brown & Los Frentones & Los Frentones \\
\hline 143 & 0133 & Almirante Brown & Taco Pozo & Taco Pozo \\
\hline 144 & $0134 \mathrm{~A}$ & General Güemes & Fortín Frías & Fuerte Esperanza \\
\hline 145 & $0134 B$ & General Güemes & El Sauzalito & El Sauzalito \\
\hline 146 & $0134 C$ & General Güemes & El Pintado & El Sauzalito \\
\hline 147 & $0134 D$ & General Güemes & Misión Nva Pompeya & Misión Nva Pompeya \\
\hline 148 & 0135 & General Güemes & Las Hacheras & Miraflores \\
\hline 149 & $0135 A$ & General Güemes & Fuerte Esperanza & Fuerte Esperanza \\
\hline 150 & $0135 B$ & General Güemes & Miraflores & Miraflores \\
\hline 151 & 0136 & General Güemes & Juan José Castelli & Juan José Castelli \\
\hline 152 & 0137 & General Güemes & Juan José Castelli & Juan José Castelli \\
\hline 153 & $0138 A$ & General Güemes & Juan José Castelli & Juan José Castelli \\
\hline 154 & $0138 B$ & General Güemes & Fortín Lavalle & Fortín Lavalle \\
\hline 155 & 0139 & General Güemes & El Espinillo & Fortín Lavalle \\
\hline
\end{tabular}

Fuente: Tribunal Electoral de la Provincia del Chaco.

En el siguiente mapa se observa la localización geográfica de los mencionados Circuitos Electorales, vimos la necesidad de ampliar el Área Metropolitana del Gran Resistencia por reunir a un grupo de Municipios importantes de la Provincia (Resistencia, Barranquera, Fontana y Puerto Vilelas), entre los cuales figura la ciudad capital donde se radica gran caudal electoral.

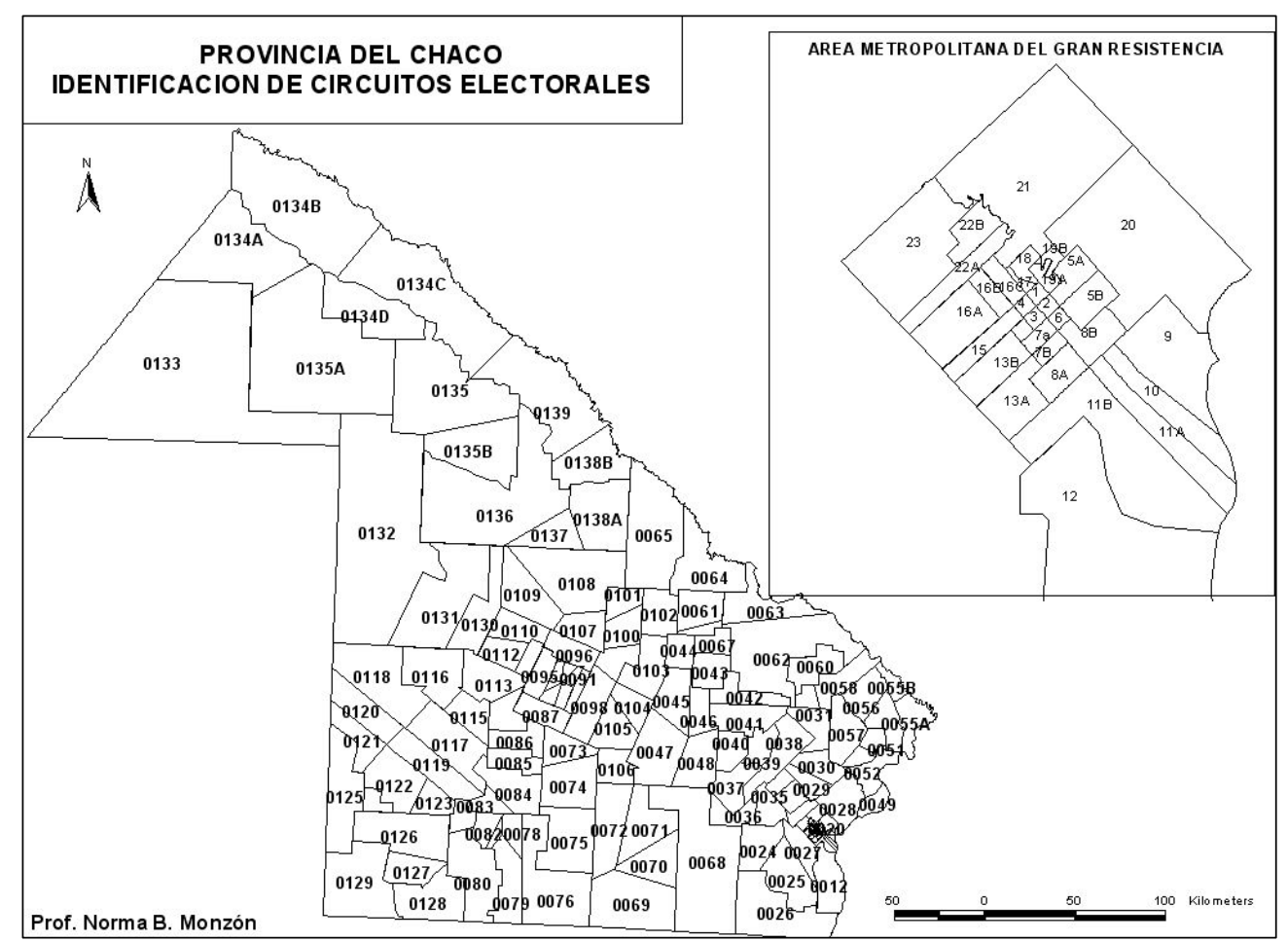

Fuente: Elaboración propia con información suministrada por la DINAE - Dirección Nacional Electoral - Ministerio del Interior - Gobierno Nacional. 


\section{EJEMPLO DE UN CASO DE ESTUDIO DE GEOGRAFÍA ELECTORAL DE LA PROVINCIA DEL CHACO. Elecciones a Gobernador 2011.}

En el año 2011 se realizaron Elecciones Nacionales de Presidente y Vice, para el caso de la Provincia del Chaco, además, ocurrieron Elecciones Legislativas - Diputados Nacionales y Provinciales -, Ejecutivas -Gobernador y Vice -, y Municipales o locales - Intendentes y Concejales En el siguiente ejemplo queremos ilustrar un caso de estudio de G.E. mostrando cartográficamente la distribución espacial por Circuito Electoral del voto para el caso del Partido Político ganador - Chaco Merece Más - en la categoría Gobernador, con valores derivados correspondiente a la participación porcentual de votos que obtuvo dicha fuerza política en relación al total de votos válidamente emitidos. Se acompaña un mapa donde se observa la distribución de los Hogares con NBI según el Censo 2010 y realizamos una breve comparación.

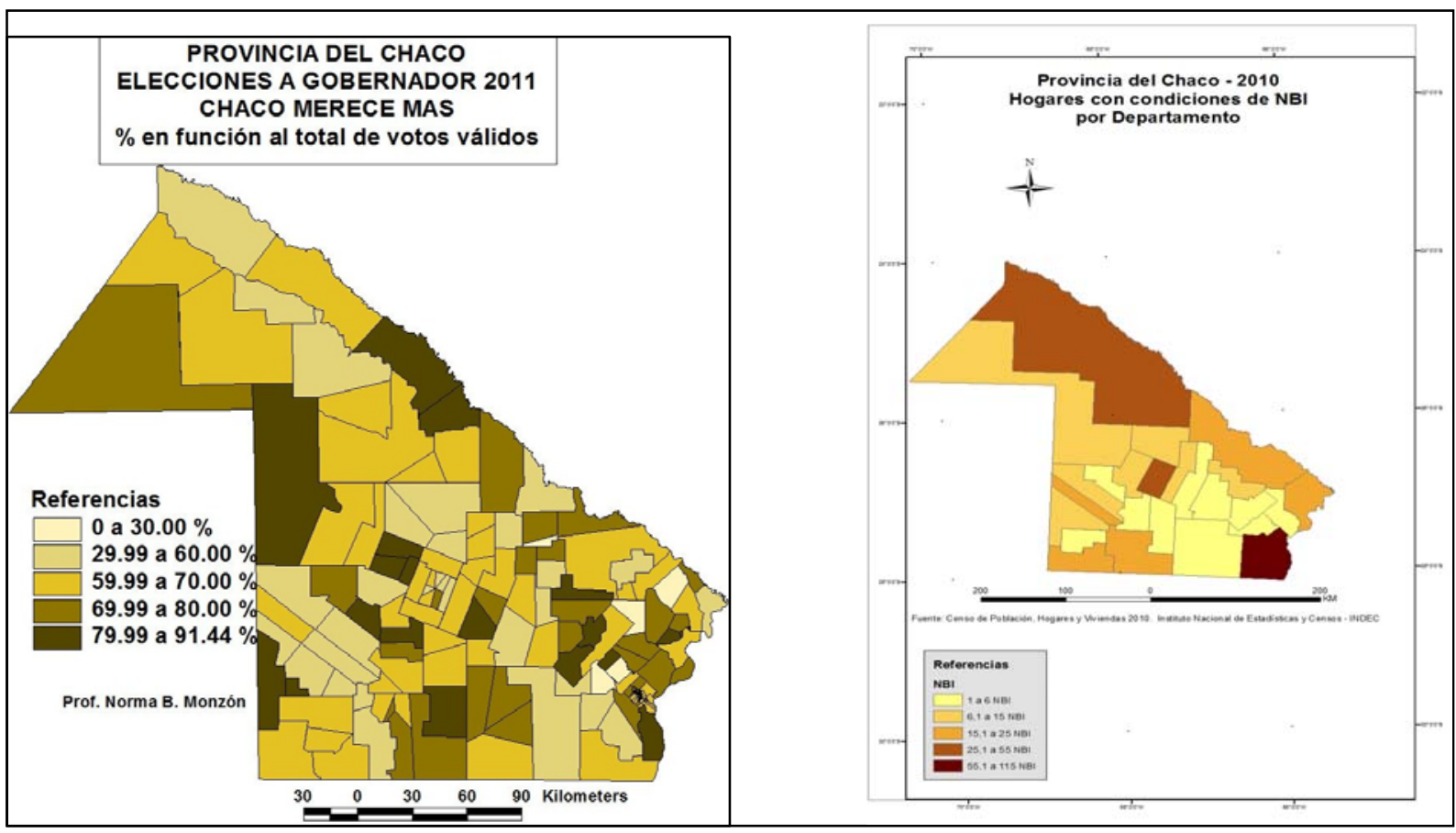

Fuente: Elaboración propia según datos del escrutinio oficial del Tribunal Electoral de la Provincia del Chaco y del Censo Nacional de Hogares, Población y Viviendas 2010 realizado por el INDEC Instituto Nacional de Estadísticas y Censo.

Según sendas representaciones, rápidamente se puede deducir una interrelación básica al observar que el voto fue muy diverso en cuanto a adherentes al Partido Político representado, y que, en general, donde ocurren menores valores de NBI (Centro, Este y Sudeste) coinciden con valores medios de votos válidos para el Frente Chaco Merece Más. Al realizar un análisis más pormenorizado, con gráficos y la inclusión de otras variables, el estudio de caso se enriquece. Solamente lo mencionamos, pues es la manera de realizar el estudio de manera completa, pero realizar ese estudio excede el objetivo del presente artículo. Solamente extraemos esta breve contribución a manera de ejemplo del método de estudio de la G.E.

\section{Dificultades durante el desarrollo de la investigación}

La principal dificultad que se presenta, y que de hecho pudimos ejemplificar en las representaciones cartográficas anteriores, es la dispar escala del espacio geográfico en la que se obtienen los datos en una variable y en otra: para el caso de los datos electorales se trabaja con Circuitos Electorales, y para el caso de los valores de NBI se obtuvo información a nivel de Departamentos. Esto ocurre de manera recurrente en las investigaciones de los geógrafos electorales.

Otra dificultad estructural es inherente a la temática misma de la G.E. Sostenemos el objetivo principal de los estudios de G.E. es analizar el comportamiento electoral, para lo cual se utilizan técnicas y métodos cualitativos y cuantitativos, pero aún no existe un método mediante el cual 
podamos acceder como insumo de información al elector de manera individual, poder conocer la manera como construyó su toma de decisión personal respecto del voto. Todo análisis se hace para el conjunto de electores que se considera en el espacio determinado de estudio.

Así mismo señalamos la escasez de marcos teóricos consensuados. La bibliografía específica sobre G.E. es relativamente escasa, considerando la amplia bibliografía que se dispone para otros aspectos de la Geografía, con el agravante de que son originadas en distintos países, se destacan México y España, pero las realidades políticas y electorales no son transmisibles de país a país, lo cual significa un continuo proceso de selección y adecuación de los trabajos considerados como antecedentes a la realidad del espacio que se desea investigar.

\section{CONCLUSION}

La G.E. es una parte de la Ciencia Geográfica cuyo desarrollo en las Universidades Nacionales argentinas es incipiente.

Anteriormente mencionamos como dificultades en la investigación a la escasa bibliografía y marco teórico consensuado, lo cual deriva en que, la mayoría de los trabajos electorales son particularmente únicos en sí mismos en cada caso.

Las características que le son propias a la G.E. la enriquecen, basadas en las técnicas utilizadas para su desarrollo que, como hemos visto, le atribuyen ser un puente entre lo cualitativo y lo cuantitativo de la Ciencia Geográfica. A su vez los insumos para la investigación provienen de fuentes de distinta índole geográfica y consecuentemente sus resultados pueden servir de base para otras investigaciones en la línea de la Geografía Social, Geografía de la Población y como aportes o sugerencias para la mejor aplicación de políticas públicas.

El territorio es estudiado en unidades espaciales posibles de análisis a gran escala, como ser los Circuitos Electorales y las fracciones y radios censales.

Por sus métodos, técnicas y resultados, estos estudios son considerados muy cercanos a la sociología, estudios sociales en general, sociología urbana y rural entre otras, siendo sus límites casi difusos entre estas ciencias.

Consideramos que es necesario que ocurra un mayor crecimiento de la G.E., pues es un aporte interesante al desarrollo de la Geografía Humana.

\section{BIBLIOGRAFÍA}

Bosque Sendra, J. 1988. Geografía Electoral. Editorial Síntesis. ISBN 84-7738-015-5. Madrid. España. $176 p$

Gómez Tagle y Valdez, 2000. La Geografía del Poder y las Elecciones en México. Plaza y Valdés Editores. ISBN 968-856-863-5 Madrid. España- 234 p.

Johnston, 1987. Diccionario de Geografía Humana. Alianza Editorial. ISBN: 9788420652245. Madrid. España. $424 \mathrm{p}$

Quesada S. 1978. La teoría de los sistemas y la Geografía Humana. En Geo crítica. Cuadernos Críticos de Geografía Humana, Universidad de Barcelona ISSN: 0210-0754 Depósito Legal: B. 9.3481976 Año III. Número: 17. España 